\title{
Utilizing diabetes registry for exploring sociodemographic and clinical characteristics of type II diabetic patients in Saudi Arabia
}

Ayla M. Tourkmani, Pharm.D, PhD, Turki J. Alharbi, MD, ADCF, Abdulaziz Bin Rsheed, MD, ADCF, Yasser K. Alotaibi, MD,FIHI.

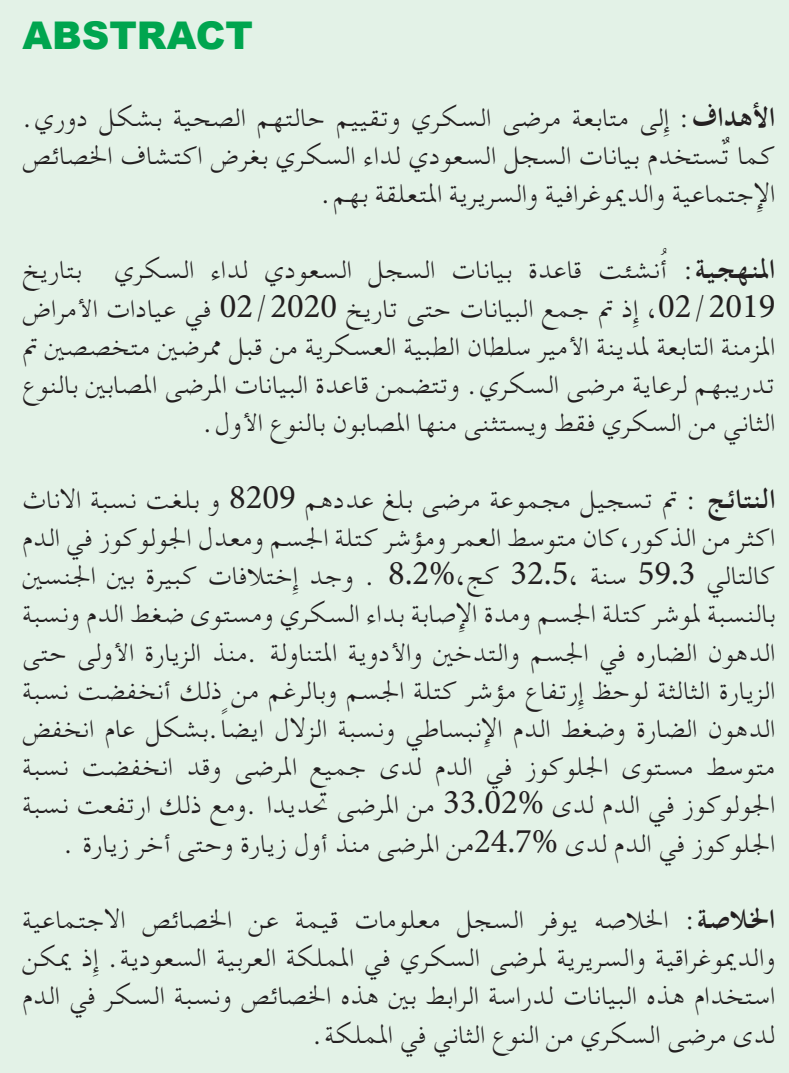

Objectives: To explore the sociodemographic and clinical characteristics as the process and outcomes of diabetic individuals.

Methods: Hospital Saudi registry at Prince Sultan Military Medical city, Chronic Illness Clinics (Family and Community Medicine), Riyadh, Saudi Arabia database was started in February 2019 and data were collected until February 2020. The data were collected by trained diabetes nurse specialists. The registry includes all patients with type II diabetes mellitus (DM) and excluded patients with type I DM.

Results: A total of 8,209 patients were enrolled in the registry with a higher proportion of females than males.
The mean age was 59.3 years, BMI $32.5 \mathrm{~kg} / \mathrm{m}^{2}$, and HBA1c levels was $8.2 \%$. Significant gender differences for BMI, duration of diabetes, blood pressure, LDL, smoking status, and medication intake. From the first to the third visit, BMI was raised; however, LDL, diastolic blood pressure, and albumin creatinine ratio were reduced. The mean HBA1c values plummeted for all patients and $33 \%$ of the patients had a reduction in the $\mathrm{HbAlc}$ levels. However, HbAlc levels increased for $24.7 \%$ of the patients' from baseline to the last visit.

Conclusion: This registry provides great insights into the sociodemographic and clinical characteristics of diabetic patients in Saudi Arabia. This registry data can be used to investigate the associations between sociodemographic or clinical characteristics and glycemic control among T2DM patients in Saudi Arabia.

Keywords: diabetes registry, sociodemographic, clinical characteristics, type II diabetes, Saudi Arabia

Saudi Med J 2021; Vol. 42 (1): 56-65 doi: $10.15537 /$ smj.2021.1.25629

From the Family and Community Medicine Department (Tourkmani, Alharbi, Bin Rsheed), Chronic Illness Center, Prince Sultan Military Medical City and from the Armed Forces Medical Services Directorate (Alotaibi), Riyadh, Kingdom of Saudi Arabia.

Received 14th September 2020. Accepted 10th December 2020.

Address correspondence and reprint request to: Dr. Ayla Tourkmani, Family and Community Medicine Department, Chronic Illness Center, Prince Sultan Military Medical City, Riyadh, Kingdom of Saudi Arabia. E-mail:aylatourkmani@gmail.com

ORCID ID: http://orcid.org/0000-0002-6060

$T$ ype 2 diabetes mellitus (T2DM) is one of the most challenging global health problems in the $21 \mathrm{st}$ century as it is a major contributor to morbidity and mortality. ${ }^{1,2}$ Quality care of diabetes patients requires an evidence-based and patient-centric approach to prevent complications, improve quality of life, and reduce disability and mortality. ${ }^{3}$ Like other countries across 
the globe, Saudi Arabia is experiencing a challenge of diabetes mellitus, where around 1792 out of 6024 (30\%) patients suffered from T2DM and generally there was a higher prevalence of diabetes in males $(34.1 \%)$ as compared to females $(27.6 \%) .{ }^{4}$ To ensure the progress of T2DM patients, it is crucial to register patients with T2DM and to monitor their characteristics and outcomes over time from a broader epidemiological perspective. ${ }^{5}$ Disease registries provide information regarding the components of the epidemiology of T2DM. ${ }^{5}$ Registries also help to improve the adherence of physicians to treatment guidelines. ${ }^{6}$

The diabetes registry is considered to monitor and evaluate patients periodically. ${ }^{7,8}$ The T2DM registry can provide data to assess the quality of treatment given to diabetic patients and make necessary modifications in the treatment plan whenever required. ${ }^{9}$ Furthermore, the T2DM registry is a valuable source to establish databases for ongoing clinical work. ${ }^{10}$ Many countries across the world have implemented their national Diabetes Registries to understand the epidemiological characteristics of the population, make decisions about the management, and to develop national guidelines. ${ }^{11-13}$ Countries such as Sweden, Denmark, Thailand, Malaysia, Singapore, and the USA have established such registries to monitor the progress of diabetic patients. ${ }^{9,11,12,14}$

Although the existing evidence provides the burden of T2DM in Saudi Arabia, there is a dearth of knowledge about how diabetic patients progress over time. ${ }^{4}$ Further, there is limited research on the combined sociodemographic and clinical characteristics of diabetic patients that can provide insights to monitor glycemic control of diabetic patients over time. Without such knowledge about various clinical and sociodemographic factors of T2DM, it is challenging to monitor such patients and provide quality of care. Thus quality monitoring is important to ensure that patients with diabetes in Saudi Arabia benefit from current improvements in care, and are treated according to the guidelines. ${ }^{15}$ To address this gap in the knowledge, King Abdulaziz City for Science and Technology (KACST) approved a large hospital-based Saudi National Diabetes Registry. ${ }^{16}$ One of the main objectives of Prince Sultan Military Medical City Diabetes registry hospital-based diabetes registry is to strengthen diabetes surveillance,

Disclosure. Authors have no conflict of interests, and the work was not supported or funded by any drug company. to manage diabetic patients, and to provide a support system to clinicians for making an evidence-based decision. This registry data can also be used to see the trends in the demographic and clinical characteristics as well as the outcomes of diabetic patients over time. We used the data from the Primary Care Center (Wazart Health Care Center, Chronic Illness Clinics (CIC)) in Prince Sultan Military Medical Services, to explore the sociodemographic and clinical characteristics and the outcomes of diabetic individuals during different visits made by them in CIC. This study was designed to address the question of what are demographic and clinical characteristics and outcomes of diabetic patients and how such patients are progressing over time? Hence, the objective of this study was to explore demographic and clinical characteristics and outcomes of diabetic patients registered from February 2019 to February 2020.

Methods. This was a retrospective cohort study where a group of patients with diabetes was followed for one year.

The diabetes registry (DR) started in February 2019 with manually manner collected data till February 2020 and data was migrated to a web-based (Oracle). This was not the implementation of a new registry rather the utilization of recent implemented registry to explore the sociodemographic and clinical characteristics of the participants. The eligibility criteria were contingent on being diagnosed with T2DM of any age or gender. However, T1DM patients and pregnant women were excluded. Data collections were started using an Excel-based application. However, after May 2019, an electronic, web-based (Oracle) under trail was used for this purpose. The status of registered patients was continuously updated throughout the frequent patients' visits/year with updates occurrence of any new complication(s) like diabetic nephropathy and renal function status. The clinic staff such as diabetes nurse specialists collected data by recording their patient's status at their regular visits. Registration was initially carried out on paper forms and was sent to the CIC-DR personnel to input into an electronic database. Following the recommendation of the Swedish National Diabetes Registry, ${ }^{17}$ the target was set as $75 \%$ of the registration rate from the total of the T2DM population. Frequent auditing was planned, which was helpful to maintain validity, reliability, and indicate the necessity for ongoing maintenance. Also, it helped for continued collaboration, inclusion, consensus, and transparency to keep diabetes stakeholders engaged and committed to using these standards of DR. 
Diabetes registry components. The component of the diabetes registry included patient's demographic data, clinical patient data, anthropometry, laboratory investigation, service utilization, foot care, eye exam, vaccine status, risk factors, and identification data of diabetes care providers. Based on monthly reporting of activities, process, and outcome measures were designed to monitor the services provided. Building upon established guidance, such as the AHRQ guidance for the development of registries to evaluate patient outcomes. ${ }^{18}$ The target for a process indicator for each patient was the availability of investigation reports during the stipulated interval (one year) or twice per year for HbAlc. Process targets included the proportion of patients with HbA1c, low-density lipoprotein (LDL), microalbuminuria, or foot examination carried out in the past one year would be designed.

Proposed outcomes. Outcome targets were set on predefined clinical outcomes. For example, the cut-off for $\mathrm{LDL}$ was $<2.5 \mathrm{mmol} / \mathrm{L}$, and for blood pressure, it was $<140 / 90 \mathrm{~mm} \mathrm{Hg}$. Likewise, normal albumin creatinine ratio was less than $1.9 \mathrm{mg} / \mathrm{mmol}$ and microalbuminuria was labeled if the ratio $(1.9 \mathrm{mg} /$ $\mathrm{mmol}$ to $33.8 \mathrm{mg} / \mathrm{mmol}$ ) and clinical albuminuria were labeled if $>33.9 \mathrm{mg} / \mathrm{mmol}$. The improvement measure based on ADA-standards were selected in our DR. Percentage of compliance to seven of 2019 ADA guideline in management of diabetes were implemented. According to these guidelines, patients were monitored for an eye examination, foot examination, vaccination status, annually of LDL, albumin/creatinine ratio, eGFR, and $\mathrm{HbA1c}$ requested at least twice/year.

Statistical analysis. This quality project included all patients follow up at the chronic illness clinic on daily basis. Results are presented as a descriptive and exploratory analysis. The results are presented as frequencies and percentages for categorical variables and mean and standard deviation (SD) for continuous variables. Chi-square and independent t-test was used to see the differences with regards to clinical and demographics variables among gender. Non-parametric tests such as the Mann-Whitney $U$ test were used where the distribution of variables was not normally distributed. A p-value of less than 0.05 was considered significant. All analysis was carried out using SPSS version 25 (IBM SPSS Statistics for Windows, version $25 .{ }^{19}$

The approval for this study was given by the Research Ethics Committee at Prince Sultan Military Medical City, Riyadh, Saudi Arabia. The original Registry data is not anonymized as it is used for clinical purposes.
However, following ethical approval for this study, our research team was given access to an anonymized version of the data that are relevant to answer our research questions.

Results. From February 2019 to February 2020, there were a total of 8,209 patients enrolled in the PSMMC Diabetes Registry with a greater proportion of females $(n=4741,57.7 \%)$ than males. The mean age of study subjects was 59.3 years and the largest proportion represented were patients at age 51-60 years old (32.5\%) followed by $61-70$ years old (27.3\%). All patients were diagnosed as Type II DM. The mean BMI of diabetic patients was $32.5 \mathrm{~kg} / \mathrm{m}^{2}$, one-third of the individuals were overweight and $32.8 \%$ categorized as obese I and II. Female patients were more obese than males however males were more overweight than females. Duration of diabetes also differed significantly among males and females; duration was longer in females $(12.5 \pm 7.6)$ as shown in Table 1 . More males compared to females were smokers $(5.8 \%)$. Blood pressure both systolic and diastolic was significantly higher among males compared to females. There was no difference between HbA1c values among gender (Table 1).

Low-density lipoprotein was significantly lower among males compared to females. eGFR value was more than 90 for $59.4 \%$ total patients. Albumin creatinine ratio did not differ among gender. The nephropathy was categorized as $35.5 \%$ patients with microalbuminuria, $6.5 \%$ with clinical albuminuria and more than half $(58 \%)$ of the patients were categorized as normal (Table 1 ).

However, microalbuminuria was common among males while clinical albuminuria did not differ among gender. Concerning the medication intake, women were taking more statin and ACEi/ ARBs compared to males however the difference was marginally significant concerning the intake of ACEi/ ARBs only as shown in Table 1. The mean HbA1c level was $8.2 \% \pm 1.7$ and at baseline, $27.9 \%$ had HbA1c of 7 and 19\% had HbA1c of more than 9 as shown in Figure 1.

The data were further categorized to assess the characteristics of patients who had baseline HbA1c $>9$ and the mean age of patients HbAlc $>9$ with was $57.8 \pm 12.2$. More than half of patients with HbAlc $>9,58.7 \%$ were female, $32.5 \%$ were of age $51-60$ and $57.4 \%$ were obese (Table 2). The mean duration of diabetes was $13.1 \pm 7.7$ and $3.1 \%$ were smokers. Mean HbAlc was $10.5 \pm 1.2$. of total $77.9 \%$ were on Statin and $63.4 \%$ were on ACEi/ ARBs Table 2.

Figure 2 demonstrates the change in BMI, LDL, HBA1c, and albumin creatinine ratio and BP by gender. 
Table 1 - Baseline and clinical characteristics of patients with respect to gender ( $\mathrm{n}=8209)$.

\begin{tabular}{|c|c|c|c|c|}
\hline Variables & $\begin{array}{l}\text { All patients } \\
(\mathrm{n}=8209)\end{array}$ & $\begin{array}{c}\text { Female } \\
(n=4741)\end{array}$ & $\begin{array}{c}\text { Male } \\
(\mathrm{n}=3468)\end{array}$ & $P$-value \\
\hline Baseline characteristics & & & & $<0.001$ \\
\hline Age & $59.3 \pm 12$ & $60.3 \pm 11.2$ & $57.9 \pm 13$ & $<0.001$ \\
\hline BMI & $32.5 \pm 6.3$ & $34.1 \pm 6.3$ & $30.5 \pm 5.6$ & \\
\hline \multicolumn{5}{|l|}{$B M I \mathrm{Kg} / \mathrm{m}^{2}$} \\
\hline Underweight $(<18.5)$ & $10 \quad(0.2)$ & $4 \quad(0.1)$ & $6 \quad(0.3)$ & $<0.001$ \\
\hline Normal $(18.5-24.9)$ & $372(7.2)$ & $123(4.2)$ & $249(11.3)$ & \\
\hline Overweight (25 to 30$)$ & $1710(33.2)$ & $757(25.6)$ & $953(43.3)$ & \\
\hline Obese 1 (30 to) & $1376(26.7)$ & $817(27.6)$ & $559(25.4)$ & \\
\hline Obese 2 (35 to) & $1144 \quad(22.2$ & $833(28.2)$ & $311(14.1)$ & \\
\hline Obese $3(>40)$ & $545(10.6)$ & $422(14.3)$ & $123(5.6)$ & \\
\hline Missing & 3052 & & & \\
\hline Duration of diabetes (years) & $12.1 \pm 7.7$ & $12.5 \pm 7.6$ & $11.6 \pm 7.8$ & $<0.001$ \\
\hline Smoker & $219(2.7)$ & $19 \quad(0.4)$ & $200(5.8)$ & $<0.001$ \\
\hline Systolic blood pressure & $127 \pm 15.8$ & $126 \pm 15.8$ & $128 \pm 15.7$ & $<0.001$ \\
\hline Diastolic blood pressure & $68 \pm 11.6$ & $65 \pm 10.7$ & $72 \pm 11.3$ & $<0.001$ \\
\hline \multicolumn{5}{|l|}{ Clinical characteristics } \\
\hline \multicolumn{5}{|l|}{ Baseline laboratory values } \\
\hline HbAlc (\%) & $8.2 \pm 1.7$ & $8.2 \pm 1.7$ & $8.2 \pm 1.7$ & 0.605 \\
\hline LDL & $2.6 \pm 0.9$ & $2.6 \pm 0.9$ & $2.5 \pm 0.9$ & 0.001 \\
\hline \multicolumn{5}{|c|}{ EGFR (total: 6899; females: 4005; males : 2894) } \\
\hline G1 $(>90)$ & $4123(59.4)$ & $2547(63.5)$ & $1576(54.4)$ & $<0.001$ \\
\hline G2 (60-89) & $2109(30.5)$ & $1123(28.1)$ & $986(34.1)$ & $<0.001$ \\
\hline $\mathrm{G} 3 \mathrm{a}(45-59)$ & $406(5.9)$ & $199(5.0)$ & $207 \quad(7.1)$ & $<0.001$ \\
\hline G3b (30-44) & $171 \quad(2.5)$ & $89(2.2)$ & $82(2.9)$ & 0.108 \\
\hline G4 (15-29) & $59(0.9)$ & $31(0.8)$ & $28 \quad(1.0)$ & 0.291 \\
\hline $\mathrm{G} 5(<15)$ & $31(0.5)$ & $16(0.4)$ & $15 \quad(0.5)$ & 0.468 \\
\hline Albumin/creatinine ratio & $1.9(0.03-1777)$ & $1.9(0.15-1737)$ & $1.9(0.03-1777)$ & 0.155 \\
\hline \multicolumn{5}{|c|}{ Albuminuria (total: 4382; females: 2554; males: 1828) } \\
\hline No albuminuria & $2542(58.0)$ & $1647(64.5)$ & $866(47.4)$ & $<0.001$ \\
\hline Micro albuminuria & $1555(35.5)$ & $741(29.0)$ & $843(46.1)$ & $<0.001$ \\
\hline Clinical albuminuria & $285(6.5)$ & $166(6.5)$ & $119(6.5)$ & 0.972 \\
\hline \multicolumn{5}{|l|}{ Medications } \\
\hline Statins & $6632(80.8)$ & $3863(81.5)$ & $2769(79.8)$ & 0.063 \\
\hline ACEi/ARBs & $4989(68.1)$ & $2915(69.0)$ & $2074(66.9)$ & 0.052 \\
\hline
\end{tabular}

BMI: body mass index, HbA1c: glycated haemoglobin, LDL: low density lipoprotein, ACEi: angiotensin converting enzyme inhibitors, ARBs: angiotensin II receptor blockers, EGFR: estimated glomerular filtration rate, ${ }^{*}$ data are reported as mean \pm standard deviation and counts with percentage, ${ }^{* *}$ comparisons were tested by Independent Samples T-test or Mann-Whitney U test for continuous variables, Pearson Chi-square test or Fisher exact test as appropriate for categorical data

Mean BMI of females raised to $34.10 \mathrm{Kg} / \mathrm{m}^{2}$ from 34.03 $\mathrm{Kg} / \mathrm{m}^{2}$ during the third visit. While the BMI of males raised to $32.05 \mathrm{Kg} / \mathrm{m}^{2}$ from 30.49 during the third visit. Further, the mean BMI increased significantly in males as opposed to females (Table 3, Figure 2). Mean LDL was reduced to $2.42 \mathrm{mmol} / \mathrm{L}$ from $2.63 \mathrm{mmol} / \mathrm{L}$ during the third visit among females and $2.36 \mathrm{mmol} / \mathrm{L}$ from $2.56 \mathrm{mmol} / \mathrm{L}$ during the third visit among males. The mean difference in reduction in LDL at third visit among males was significantly reduced to $2.45(0.85)$ compared to $2.52(0.85)$ among females with a p-value $<0.01$. Mean HbA1c increased to $8.26 \%$ from $8.20 \%$ during the third visit among females while in males mean $\mathrm{HbA} 1 \mathrm{c}$ reduced to $8.03 \%$ from $8.18 \%$ during the third visit (Table 3, Figure 2). The mean difference in HbA1c levels did not differ among gender (male: 7.94 (1.58) versus female: 7.98 (1.62); $p=0.274)$. The mean Albumin creatinine ratio was reduced to 12.01 from 12.36 during the third visit among females and 10.08 from 12.22 during the third visit among males as shown in Figure 2. There was no difference in the reduction of Albumin/Creatinine ratio at third visit among gender with a p-value for Man-Whitney U test 0.593. Mean diastolic blood pressure was reduced to $64.70 \mathrm{~mm}$ $\mathrm{Hg}$ from $65.13 \mathrm{~mm} \mathrm{Hg}$ among females during their third visit and it reduces in males to $69.82 \mathrm{~mm} \mathrm{Hg}$ 


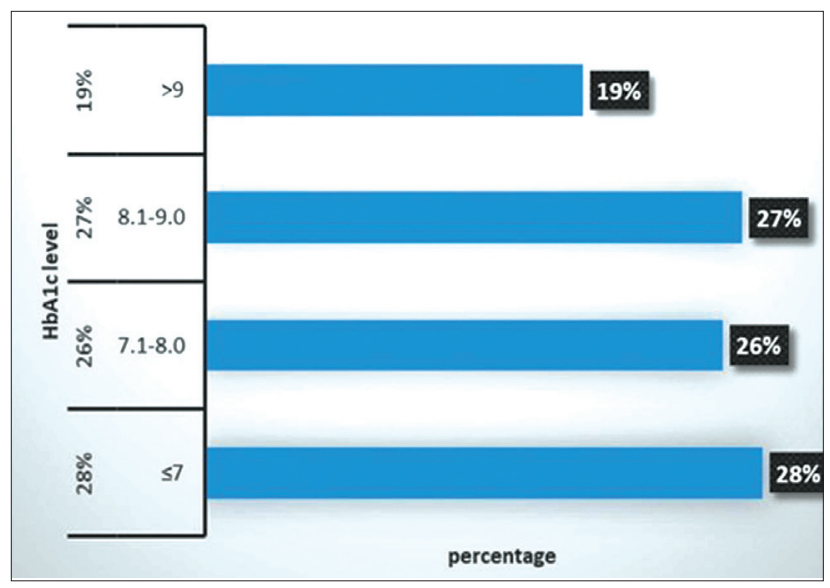

Figure 1 - Distribution of diabetes mellitus (DM) patient's HbA1c levels at time of enrolment in the DM registry.

Table 2 - Characteristics of patients with HbAlc >9\% at baseline.

\begin{tabular}{|c|c|c|}
\hline Variables & $\begin{array}{l}\text { All patients } \\
(\mathrm{n}=8209)\end{array}$ & $\begin{array}{c}\text { HbA1c } \geq 9 \% \\
\text { at baseline } \\
(n=1543)\end{array}$ \\
\hline Age & $59.3 \pm 12$ & $57.8 \pm 12.2$ \\
\hline Female & $4741(57.8)$ & $906(58.7)$ \\
\hline Male & $3468(45.2)$ & $637(41.3)$ \\
\hline \multicolumn{3}{|l|}{ Age categories (years) } \\
\hline$\leq 20$ & $7 \quad(0.1)$ & $2(0.1)$ \\
\hline $21-30$ & $68(0.8)$ & $18(1.2)$ \\
\hline $31-40$ & $420 \quad(5.1)$ & $100(6.5)$ \\
\hline $41-50$ & $1388(16.9)$ & $310(20.1)$ \\
\hline $51-60$ & $2672(32.5)$ & $502(32.5)$ \\
\hline $61-70$ & $2244(27.3)$ & $396(25.7)$ \\
\hline$>70$ & $1410(17.2)$ & $215(13.9)$ \\
\hline BMI & $32.5 \pm 6.3$ & $32.2 \pm 6.3$ \\
\hline \multirow{2}{*}{\multicolumn{3}{|c|}{$\begin{array}{l}\text { BMI categories } \mathrm{Kg} / \mathrm{m}^{2} \text { (out of } \\
5157 \text { of all patients and } 1400 \\
\text { of those with } \mathrm{HbA1c} \geq 9 \% \text { at } \\
\text { baseline) }\end{array}$}} \\
\hline & & \\
\hline Underweight $(<18.5)$ & $10(0.2)$ & $3(0.2)$ \\
\hline Normal (18.5-24.9) & $372(7.2)$ & 124 \\
\hline Overweight ( 25 to 30$)$ & $1710(33.2)$ & $469(33.5)$ \\
\hline Obese $1(30$ to $<35)$ & $1376(26.7)$ & $342(24.4)$ \\
\hline Obese $2(35$ to $<40)$ & $1144(22.2)$ & $331(23.6)$ \\
\hline Obese $3(>40)$ & $545(10.6)$ & $131(9.4)$ \\
\hline Duration of diabetes (years) & $12.1 \pm 7.7$ & $13.1 \pm 7.7$ \\
\hline Smoker & $219(2.7)$ & $70 \quad(3.1)$ \\
\hline Systolic blood pressure & $127 \pm 15.8$ & $127 \pm 16.2$ \\
\hline Diastolic blood pressure & $68 \pm 11.6$ & $68.6 \pm 11.7$ \\
\hline \multicolumn{3}{|l|}{ Baseline laboratory values } \\
\hline HbA1c (\%) & $8.2 \pm 1.7$ & $10.5 \pm 1.2$ \\
\hline LDL & $2.6 \pm 0.9$ & $2.8 \pm 1$ \\
\hline Albumin/creatinine ratio & $1.9(0.03-1777)$ & $1.9(0.01-1737)$ \\
\hline \multicolumn{3}{|l|}{ Medications } \\
\hline Statins & $6632(80.8)$ & $1201(77.9)$ \\
\hline ACEi/ ARBs & $4989(68.1)$ & $978(63.4)$ \\
\hline
\end{tabular}

BMI: body mass index, HbA1c: glycated hemoglobin, LDL: low density lipoprotein, ACEi: angiotensin converting enzyme inhibitors, ARBs: angiotensin II receptor blockers. ${ }^{*}$ Data are reported as mean \pm standard deviation and counts with percentage. from $72.42 \mathrm{~mm} \mathrm{Hg}$ among males during their third visit (Table 3, Figure 2). While systolic blood pressure did not vary much among both females and males. The mean difference in reduction in diastolic blood pressure at third visit among females was significantly reduced to 63.50 (10.51) compared to 70.39 (11.39) among males $(p<0.001)$. Likewise, the mean difference in reduction in systolic blood pressure at the third visit among females was significantly reduced to 123.67 (15.24) compared to 125.57 (15.36) among males $(p<0.001)$ (Table 3, Figure 2).

The patient's baseline and their last $\mathrm{HbAlc}$ reports were measured to determine the changes in HbAlc. A total of 5689 patients had their baseline line and last HbA1c levels. Of these 1879 (33.02\%) patients had a reduction in HbA1c levels at their last visit. Among these patients with improved HbAlc levels at their last visit $559(29.7 \%)$ achieved the targeted HbA1c level that is $<7 \%$. Moreover, $24.6 \%$ patients had an increased in their HbAlc at their last visit compared to the baseline (Figure 3). Almost all of our patients were meeting the recommendations for annual HBA1c and LDL check-ups with almost equal distribution for males and females, and $82.65 \%$ and $86.31 \%$ were compliant for the eye and foot examination respectively (data not shown).

Discussion. Findings from a large hospital-based registry for T2DM in Saudi Arabia revealed that greater proportions of individuals enrolled in the registry were females than males. These findings are consistent with the Malaysian registry where $55.9 \%$ of the patients were females than $44.1 \%$ of males. ${ }^{20}$ However, our findings contradict the findings from the Saudi National Diabetes Registry (SNDR) electronic database, where they found a greater proportion of males $(51.2 \%)$ compared to females $(48.8 \%){ }^{21}$ Similarly, Aronson et $\mathrm{al}^{20}$ explored the Diabetes Registry database in Canada, where they found a higher proportion of males than females. The differences in findings can be explained by differences in risk factors between countries such as stress, other comorbidities, family history, and pregnancy leading to gestational diabetes, which in turn could progress to type II diabetes. ${ }^{22,23}$ However, we did not have data on gestational diabetes to support this finding. Regarding age distribution, the mean age of our diabetes registry individuals was 59.3 years, and around one-third were in the 51-60 years old category (32.5\%) followed by $61-70$ years $(27.3 \%)$. The mean BMI of diabetic patients was $32.5 \mathrm{~kg} / \mathrm{m}^{2}$, one-third of the individuals were overweight and $32.8 \%$ categorized as obese I and II as per the criteria of the World Health Organization (WHO) 
definition. These findings are similar to the Malaysian as well as the National Diabetes Registry in Saudi Arabia registry where the mean age of T2DM patients across the samples was 60 years old and the mean duration of diabetes was 13.55 years $( \pm 8.14)$ years. ${ }^{20}$ The combined accumulation of over-age risk factors due to lifestyle changes, obesity, dietary changes, stressful life, and lack of physical activity may explain these findings. ${ }^{24,25}$ In the current study, such variables were not examined but could explain the results of higher mean age of diabetic patients as well as the consistency with other studies regarding these findings. Besides, our Diabetes Registry database showed that the mean HbAlc level was $8.2 \% \pm 1.7$, which was comparable to the results from National Diabetes Registry in Malaysia (mean HbA1c: $8.1 \%) .{ }^{20}$ On the other hand, our findings differ from the findings of the Canadian diabetes registry database, where HbAlc levels ranged from $6.8 \%$ to $7.1 \% .{ }^{20}$ The
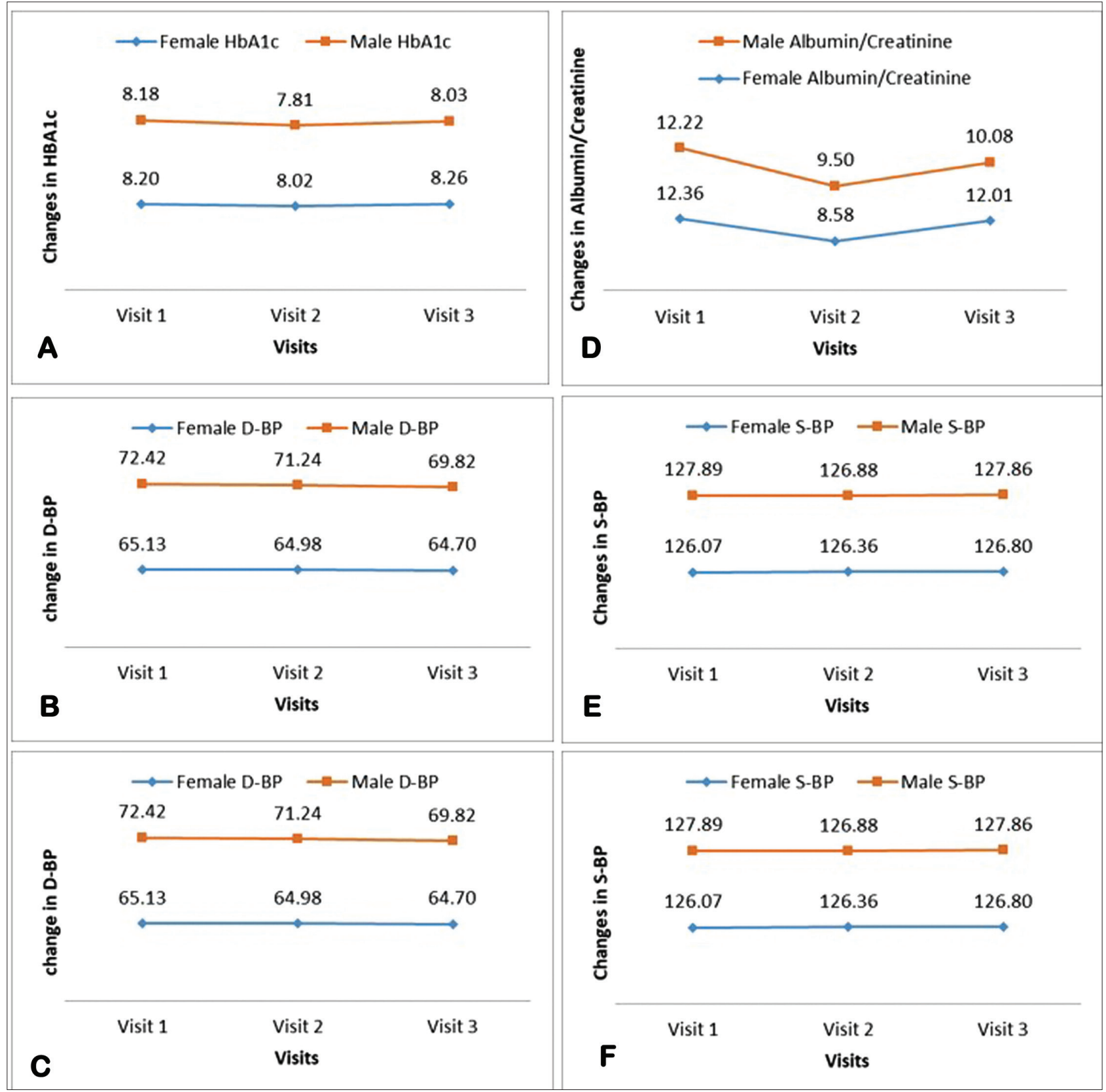

Figure 2 - Change in BMI, LDL, HbA1c, albumin creatinine ratio and BP among males and females. 
Table 3 - Mean differences in BMI, LDL, HbAlc, albumin creatinine ratio and $\mathrm{BP}$ among males and females.

\begin{tabular}{|c|c|c|c|}
\hline Mean differences & Female & Male & $P$-value \\
\hline Mean diff in BMI & $33.80 \quad(6.16)$ & $30.69(10.87)$ & $<0.001$ \\
\hline Mean diff in LDL & $2.52 \quad(0.85)$ & $2.45 \quad(0.85)$ & 0.001 \\
\hline Mean diff in $\mathrm{HbA} 1 \mathrm{c}$ & $7.98 \quad(1.62)$ & $7.94 \quad(1.58)$ & 0.272 \\
\hline Mean diff in DBP & $63.50(10.51)$ & $70.39(11.39)$ & $<0.001$ \\
\hline Mean diff in SBP & $123.67(15.24)$ & $125.57(15.36)$ & $<0.001$ \\
\hline Mean diff in AC ratio* & 2793.10 & 2770.13 & 0.593 \\
\hline \multicolumn{4}{|c|}{$\begin{array}{l}\text { *mean rank is reported, BMI: body mass index, LDL: low density } \\
\text { lipoprotein, HbA1c: glycated hemoglobin, DBP - diastolic blood } \\
\text { pressure, SBP- systolic blood pressure, AC: albumin creatinine ratio }\end{array}$} \\
\hline
\end{tabular}

potential discrepancies in the health care system and the obligation of the patient to track glycemic regulation should collectively explain these results. For instance, higher HbA1c levels in Saudi Arabia and Malaysia may be due to poor diabetic patient compliance or lack of monitoring for glycemic control and behavior factors such as lack of exercise. ${ }^{26-28}$ In comparison, patients in Canada have lower levels of HbA1c, which may suggest that their health care providers may follow the recommended guidelines vigilantly to improve glycemic control in diabetic patients. ${ }^{29}$ We found that in the current registry 28\% had HbAlc $<7$ and 26\% had $\mathrm{HbAlc}$ from 7.1-8. The reason for considering the HbAlc from 7.1-8 was due to less stringent HbAlc goal for some elderly patients as per American Diabetes Association (ADA) recommendation, ${ }^{30}$ and our results showed that $19 \%$ of the patients had $\mathrm{HbA} 1 \mathrm{c}>9$ at baseline and $14 \%$ had a refractory persistent elevation of HbA1c $>9$ for more than 6 months. The patients who had baseline HbA1c $>9$ were older, mostly females and more than half of them were obese. These patients had a longer mean duration of diabetes, more than half of them were taking medications but surprisingly very few of them were smokers. These findings are consistent with other studies across the world, which have found that people with a longer duration of diabetes and with higher BMI values have poor glycemic control. ${ }^{31}$ The relationship of taking medications with higher levels of $\mathrm{HbA1c}$ is consistent with the results of other studies. ${ }^{31-33}$ Collectively, these results may be explained by the fact that diabetes normally continues to deteriorate over time, as well as by the fact that existing treatment regimens may not be adequately implemented to achieve the goals of care as revealed in the literature. ${ }^{34,35}$

Diabetic nephropathy was another major clinical outcome predictor derived from this diabetes registry.

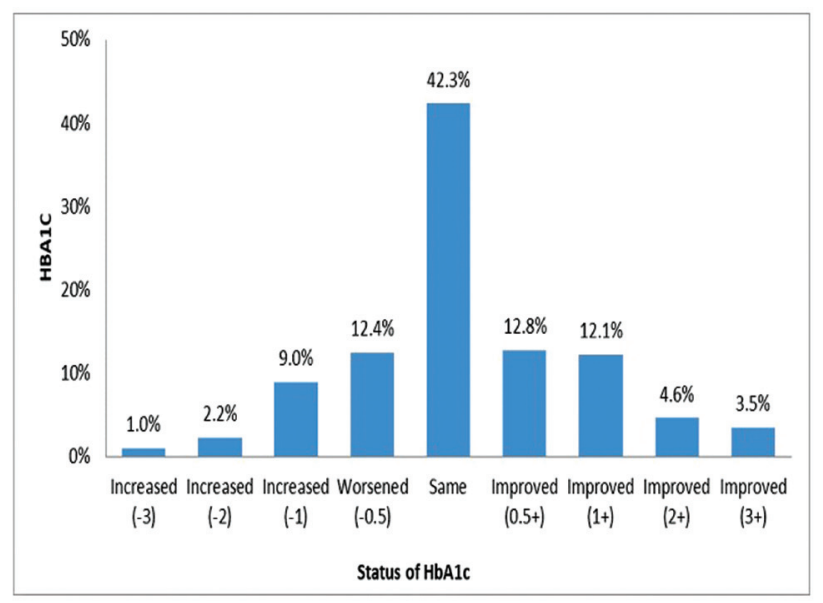

Figure 3 - Status of HbA1c: glycated hemoglobin compared to the baseline measures $(\mathrm{N}=5689)$.

The Diabetes Registry's results measured and classified nephropathy as common in $35.7 \%$ of patients with microalbuminuria, $6.5 \%$ with clinical albuminuria, and approximately $58.1 \%$ of patients not performed albumin to creatinine ratio (ACR) test as recommendation. In 2014, Al-Rubeaan et a ${ }^{36}$ selected data from the National Diabetes Registry to test Diabetic Nephropathy among 54,670 diabetes patients. In their study, the authors found that the overall incidence of diabetic nephropathy was $10.8 \%$, with $1.2 \%$ of diabetic patients suffering from microalbuminuria, $8.1 \%$ from macroalbuminuria, and $1.5 \%$ from end-stage renal disease. ${ }^{36}$ In addition, the examination of renal function revealed that $57.34 \%$ of patients had eGFR $>90$ and $82 \%$ of patients had blood pressure greater than $140 / 90$. Besides, the mean nephropathy rate was $19.7 \pm 0.4$ and $47 \pm 0.6$ without microalbuminuria among 10590 T2DM patients in the LMC diabetes registry in Canada. ${ }^{37}$

We found significant gender differences for BMI, duration of diabetes, blood pressure, LDL, smoking status, and medication intakes. However, we did not find any gender differences in $\mathrm{HbAlc}$ and clinical albuminuria. Our finding regarding women being more obese than men is consistent with findings from other countries. ${ }^{38}$ This finding can be explained by the fact that a woman becomes pregnant and give birth to children. Therefore, pregnancy also may play a role in the tendency for women to be more obese, hypertensive, and diabetic than men. The overall effect of the metabolic changes in late normal pregnancy is diabetogenic and characterized by insulin resistance. ${ }^{39}$ In addition, women might tend to stay more at home and exercise less than men due to their busy schedules 
for work and household chores. In this research, which is similar to the current literature, we found males to be more smokers than females, suggesting that males are typically more smokers than females regardless of any disease status, indicating disparities in the behavior of females and males. ${ }^{40,41}$ However, these explanations need to be explored further in future studies. The findings showed that women had higher levels of LDL and had diabetes for a longer period than men, which is consistent with other studies. ${ }^{40}$ The mechanisms leading to a greater augmentation of these factors among diabetic women compared with that of diabetic men have remained largely unknown. One of the possible explanations of higher LDL levels among women in our data could be due to the reason for the higher proportion of obese I (27.6\%), II (28.4\%), and III (14.3\%) categories. There is an enormous amount of premise on association between obesity and abnormalities in lipid metabolism mainly among women. ${ }^{41-44}$

The data revealed, as predicted, that both systolic and diastolic blood pressure were statistically but clinically negligible higher among males compared to females, showing that no significant difference between males and females was observed. Larger populationbased research and meta-analysis indicate that across middle age, males typically have a higher blood pressure than females. ${ }^{45}$ However, the mechanisms responsible for hypertension among males are complicated by comorbid conditions of obesity and type II diabetes, both of which lead to increases in blood pressure ${ }^{46}$ and need to be explored further in the Saudi Population. These suboptimal controls of BP, lipid profiles, and BMI, which are differed by gender must be highlighted as these sub-optimally controlled clinical parameters of T2DM patients can cause large health care and economic burden for the government of Saudi. However, the registry data revealed that a greater proportion of our population $(60 \%)$ was found to have LDL levels below $2.6 \mathrm{mmol} / \mathrm{L}$ as one of the important clinical outcome indicators, which might indirectly indicate good compliance with medications. This is in contrast to the findings from the Singapore Diabetes registry the results of where only $35.5 \%$ to $52 \%$ of the diabetic patients had LDL levels $<2.6 \mathrm{mmol} / \mathrm{l}$ in 2 connective years 2007 and 2008). ${ }^{47}$

We observed that overall BMI increased between males and females from the first to the third visit, while LDL, diastolic blood pressure, and albumin creatinine ratio decreased between the 2 sexes from the first to the third visit while investigating the patterns of different clinical features of diabetic patients over different visits. Our results on uncontrolled BMI are consistent with findings from a Malaysian study. ${ }^{48}$ Overall, these findings implicitly suggest that T2DM patients are compliant with the drugs and are committed to achieving glycemic regulation. However, considering the increase in BMI over different visits, for these T2DM patients, multiple exercise programs with strict weight control may be required. Moreover, normalization of insulin sensitivity and blood glucose levels are seen in T2DM patients who achieve pronounced weight loss. ${ }^{49}$ For example, the same study revealed that obese patients had worse glycemic control and lipid profiles and higher BP compared than non-obese patients, irrespective of the age. ${ }^{49}$ Therefore, obesity prevention and weight reduction is urgent and should include the cooperation of public health institutions, the school systems, and the private sector in fighting the growing obesity amongst type 2 diabetic patients.

Using registry data, we also looked at the adherence of our doctors to some clinical indicators process as per American Diabetes Association Recommendation. We found that almost all of our patients were meeting the recommendations for annual HBAlc and LDL check-ups with almost equal distribution for males and females, and a substantial proportion of the physicians requesting the lab investigations were compliant for the eye and foot examination by $82.65 \%$ and $86.31 \%$, which were higher when compared to the data from Malaysian diabetes registry. ${ }^{20}$ More specifically, the data from the Malaysian Diabetes Registry shows the proportion of fundus examination as $44 \%$ and foot examination at least once annually only was $73 \% .^{20}$ Moreover, in our diabetes registry, the albumin creatinine ratio requested annually was shown as $71.43 \%$, which was higher when compared to the National Diabetes Registry Malaysia $(64.7 \%) .{ }^{20}$ In Singapore, the diabetes registry measured the process indicator in chronic care, which showed that the rates of nephropathy assessment every 15 months was $87 \%$. However, in the Singapore Diabetes registry, annual foot screening was measured but this neither showed a record of the diabetic foot ulcer scale (DFS) nor any visit to a Podiatrist. ${ }^{47}$ In addition, $68.1 \%$ and $80.8 \%$ of T2DM patients reported using medications such as ACE-inhibitors and statins compared to $49 \%$ of the patients who used ACE-inhibitors as antihypertensive medications and $62.3 \%$ using statins for dyslipidemia management. The possible reasons for such subtle differences could be due to limited access to services such as the availability of a podiatrist or limited knowledge of DM patients about the importance of such screening procedures.

Study limitations. One of the potential strengths of this study was the large database from the ongoing 
registry with a greater sample size to describe the characteristics of the T2DM people. In addition, the data in this study were collected both directly from the patients' records, and by an active approach of patients in case of missing or outdated variables as well. With this procedure, it seems plausible that the underrepresentation of patients was limited. Characteristics of patients such as mean age, gender, glycemic control, and the known duration of the disease were comparable to other recent investigations in general practice. Thus, it is highly likely that our findings were representative of general practice in Saudi Arabia. However, this study has some caveats that need to be addressed in the future. One of the main limitations was we could not study the association between various sociodemographic or clinical characteristics with glycemic control among T2DM patients. In addition, we neither had a pharamco-epidemiological database nor had data on medication history such as the use of insulin or oral medications used for diabetes. Similarly, detailed information was not available regarding other diabetesrelated complications such as diabetic neuropathy and retinopathy, this can cause a delay in the progression of the diabetes registry framework. Furthermore, the privation of referral to the emergency department and changes in medical status, living conditions, and mortality rate are a few of the essential parameters that should be considered to modify the diabetes registry design. Lastly, since it was a retrospective study therefore had caveat of missing data, which might have missed information on important parameters or observations.

In conclusion, a greater proportion of patients were found to be overweight and obese in the registry results, there is a need to establish tailored therapies for such patients. The majority of the patients were found to be adherent with curative aspects of diabetes management as per American Diabetes Association Recommendation, however, there is a need to divert focus on the preventive aspects such as being vaccinated against flu or pneumonia. Higher mean HBA1c values indicate inadequate glucose level control. This registry data can be used in the future to investigate the associations between different sociodemographic or clinical features and glycemic regulation among patients with T2DM. To develop a gender-based care and management strategy and concentrated prevention measures, more studies are needed to understand the variations in different clinical indicators of DM between genders as well as across countries.

Acknowledgment. The authors would like to thank the Quality Department at the Medical Services Directorate for their guidance and support towards the development of the first diabetes registry in the Military Hospital in primary care centers. We are indebted to the General Executive Director of Prince Sultan Military Medical City, Major General Dr. Saud Al Shlash for his tremendous support. And to all the clinicians including physicians, clinical pharmacists, nurses, diabetic educators, dietitians, social workers, diabetes registry coordinators (Anwar Alotaibi and Amal Alotaibi), Ms Reuof Bushnag from the Wazarat Health Care Centers, Dr. Azzam AlOtabi, who is in charge of Wazarat Health Care Center and Deputy Dr. Mohammed AbdulMogith for their support to establish this program. In addition, we acknowledge translation units for their help in translating the abstract. Our thanks expand to the Information Technology unit in Prince Sultan Military Medical City, Mrs. Wafa Al Bablal, Salman AlOtaibi, Mr Peer Shariff, and Mr. Fedda Hussein for their dedication to establishing the new electronic diabetes registry program.

\section{References}

1. Ginter E, Simko V. Type 2 diabetes mellitus, pandemic in 21 st century. Adv Exp Med Biol 2012; 771: 42-50.

2. Muilwijk M, Ho F, Waddell H, Sillars A, Welsh P, Iliodromiti $S$, et al. Contribution of type 2 diabetes to all-cause mortality, cardiovascular disease incidence and cancer incidence in white Europeans and South Asians: findings from the UK Biobank population-based cohort study. BMJ Open Diabetes Res Care 2019; 7: e000765.

3. Heng BH, Sun Y, Cheah JT, Jong M. The Singapore national healthcare group diabetes registry-Descriptive epidemiology of type 2 diabetes mellitus. Ann Acad Med Singap 2010; 39: 348-352.

4. Alqurashi KA, Aljabri KS, Bokhari SA. Prevalence of diabetes mellitus in a Saudi community. Ann Saudi Med 2011; 31: 19-23.

5. Sreedharan J. The need to establish local Diabetes Mellitus registries. Nepal J Epidemiol 2016; 6: 551-552.

6. Gabbay RA, Khan L, Peterson KL. Critical features for a successful implementation of a diabetes registry. Diabetes Technol Ther 2005; 7: 958-967.

7. American Diabetes Association. Diagnosis and classification of diabetes mellitus. Diabetes Care 2013; 36 Suppl 1: S67-S74.

8. Diagnosis and Classification of Diabetes Mellitus. Diabetes Care 2014; 37 (Supplement 1): S81-S90.

9. Jørgensen ME, Kristensen JK, Husted GR, Cerqueira C, Rossing P. The Danish adult diabetes registry. Clin Epidemiol 2016; 8: 429-434.

10. Gudbjornsdottir S, Cederholm J, Nilsson PM, Eliasson B. The National Diabetes Register in Sweden: an implementation of the St. Vincent Declaration for Quality Improvement in Diabetes Care. Diabetes Care 2003; 26: 1270-1276.

11. Rawdaree P, Ngarmukos C, Deerochanawong C, Suwanwalaikorn S, Chetthakul T, Krittiyawong S, et al. Thailand diabetes registry (TDR) project: clinical status and long term vascular complications in diabetic patients. $J$ Med Assoc Thai 2006; 89 (Suppl 1): S1-S9.

12. Chamany S, Silver LD, Bassett MT, Driver CR, Berger DK, Neuhaus CE, et al. Tracking diabetes: New York City's A1C Registry. The Milbank Quarterly 2009; 87: 547-570.

13. Toh MP, Leong HS, Lim BK. Development of a diabetes registry to improve quality of care in the National Healthcare Group in Singapore. Ann Acad Med Singap 2009; 38: 546-546.

14. Elfgren I, Tornvall E, Grodzinsky E. The processes of implementation of the diabetes register in primary health care. Int J Qual Health Care 2012; 24: 419-424. 
15. Al-Faris EA. Guidelines for the management of diabetic patients in the health centers of Saudi Arabia. J Family Community Med 1997; 4: 12-23.

16. Al-Rubeaan KA, Youssef AM, Subhani SN, Ahmad NA, Al-Sharqawi AH, Ibrahim HM. A Web-based interactive diabetes registry for health care management and planning in Saudi Arabia. J Med Internet Res 2013; 15: e202.

17. Hallgren Elfgren IM, Tornvall E, Grodzinsky E. The process of implementation of the diabetes register in Primary Health Care. Int J Qual Health Care 2012; 24: 419-424.

18. Gliklich RE, Dreyer NA, Leavy MB, editors. Registries for Evaluating Patient Outcomes: A User's Guide [Internet]. 3rd ed. Rockville (MD): Agency for Healthcare Research and Quality (US); 2014 Apr. Report No.: 13(14)-EHC111.

19. Field A. Discovering statistics using IBM SPSS statistics: North American edition. 5th ed. Los Angeles (CA): SAGE Publications 2017.

20. CW T. Outcomes of Diabetes Care in Primary Care Services at Malaysia: A Retrospective Analysis of National Diabetes Registry (NDR) Database.

21. Al-Rubeaan K, Abu El-Asrar AM, Youssef AM, Subhani SN, Ahmad NA, Al-Sharqawi AH, et al. Diabetic retinopathy and its risk factors in a society with a type 2 diabetes epidemic: a Saudi National Diabetes Registry-based study. Acta Ophthalmol 2015; 93: e140-e147.

22. Thibault V, Bélanger M, LeBlanc E, Babin L, Halpine S, Greene B, Mancuso M. Factors that could explain the increasing prevalence of type 2 diabetes among adults in a Canadian province: a critical review and analysis. Diabetol Metab Syndr 2016; 8: 71.

23. Fareed M, Salam N, Khoja AT, Mahmoud AM, Ahamed M. Life style related risk factors of type 2 diabetes mellitus and its increased prevalence in Saudi Arabia: a brief review. International Journal of Medical Research Health Sciences 2017; 6: 125-132.

24. Kolb H, Martin S. Environmental/lifestyle factors in the pathogenesis and prevention of type 2 diabetes. BMC Medicine 2017; 15: 131 .

25. Chong S, Ding D, Byun R, Comino E, Bauman A, Jalaludin B. Lifestyle changes after a diagnosis of type 2 diabetes. Diabetes Spectrum 2017; 30: 43-50.

26. Alzaheb RA, Altemani AH. The prevalence and determinants of poor glycemic control among adults with type 2 diabetes mellitus in Saudi Arabia. Diabetes Metab Syndr Obes 2018; 11 : 15-21.

27. Alramadan MJ, Magliano DJ, Almigbal TH, Batais MA, Afroz A, Alramadhan HJ, Mahfoud WF, Alragas AM, Billah B. Glycaemic control for people with type 2 diabetes in Saudi Arabia - an urgent need for a review of management plan. BMC Endocr Disord 2018; 18: 62.

28. Balasubramaniam S, Lim SL, Goh LH, Subramaniam S, Tangiisuran B. Evaluation of illness perceptions and their associations with glycaemic control, medication adherence and chronic kidney disease in type 2 diabetes mellitus patients in Malaysia. Diabetes Metab Syndr 2019; 13: 2585-2591.

29. Cheng AY, Lau DC. The Canadian Diabetes Association 2013 clinical practice guidelines-raising the bar and setting higher standards! Can J Diabetes 2013; 37: 137-138.

30. Tay TL, Foo JP, Tan E, Chen R, Khoo J, Soh SB, Au V, Cho LW. HbA1c may not be a sensitive determinant of diabetic status in the elderly. Diabetes Res Clin Pract 2011; 92: e31-e33.

31. Goudswaard AN, Stolk RP, Zuithoff P, Rutten GE. Patient characteristics do not predict poor glycaemic control in type 2 diabetes patients treated in primary care. Eur J Epidemiol 2004; 19: 541-545.
32. Harmel AP, Ryan D, Thompson R. Glycohemoglobin assessment program: glycated hemoglobin and epidemiologic variables in patients with type 2 diabetes. Endocr Pract 2002; 8: 184-190.

33. Shorr RI, Franse LV, Resnick HE, Di Bari M, Johnson KC, Pahor M. Glycemic control of older adults with type 2 diabetes: findings from the Third National Health and Nutrition Examination Survey, 1988-1994. J Am Geriatr Soc 2000; 48: 264-267.

34. Trikkalinou A, Papazafiropoulou AK, Melidonis A. Type 2 diabetes and quality of life. World J Diabetes 2017; 8: 120-129.

35. Boulle P, Kehlenbrink S, Smith J, Beran D, Jobanputra $\mathrm{K}$. Challenges associated with providing diabetes care in humanitarian settings. The Lancet Diabetes \& Endocrinology 2019; 7: 648-656.

36. Al-Rubeaan K, Youssef AM, Subhani SN, Ahmad NA, Al-Sharqawi AH, Al-Mutlaq HM, David SK, AlNaqeb D. Diabetic nephropathy and its risk factors in a society with a type 2 diabetes epidemic: a Saudi National Diabetes Registry-based study. PLoS One 2014; 9: e88956.

37. Aronson R, Orzech N, Ye C, Goldenberg R, Brown V. Specialistled diabetes registries and predictors of poor glycemic control in type 2 diabetes: Insights into the functionally refractory patient from the LMC Diabetes Registry database. J Diabetes 2016; 8: 76-85.

38. Lasky D, Becerra E, Boto W, Otim M, Ntambi J. Obesity and gender differences in the risk of type 2 diabetes mellitus in Uganda. Nutrition 2002; 18: 417-421.

39. Zeman FJ. Clinical nutrition and dietetics. Dunfermline (UK): Collamore Press Inc.; 1983.

40. Juutilainen A, Kortelainen S, Lehto S, Rönnemaa T, Pyörälä $\mathrm{K}$, Laakso M. Gender difference in the impact of type 2 diabetes on coronary heart disease risk. Diabetes Care 2004; 27: 2898-2904.

41. Howard BV, Ruotolo G, Robbins DC. Obesity and dyslipidemia. Endocrinol Metab Clin North Am 2003; 32: 855-867.

42. Misra A, Shrivastava U. Obesity and dyslipidemia in South Asians. Nutrients 2013; 5: 2708-2733.

43. Link JC, Reue K. Genetic Basis for Sex Differences in Obesity and Lipid Metabolism. Annu Rev Nutr 2017; 37: 225-245.

44. Haider K, Rehman K, Akash MSH. Impaired lipid metabolism in metabolic disorders. Endocrine disrupting chemicals-induced metabolic disorders and treatment strategies. Manhattan (UK): Springer; 2020. p. 83-94.

45. Staessen J, Fagard R, Lijnen P, Thijs L, van Hoof R, Amery A. Reference values for ambulatory blood pressure: a meta-analysis. J Hypertens Suppl 1990; 8: S57-S64.

46. Burt VL, Whelton P, Roccella EJ, Brown C, Cutler JA, Higgins M, et al. Prevalence of hypertension in the US adult population: results from the Third National Health and Nutrition Examination Survey, 1988-1991. Hypertension 1995; 25: 305-313.

47. Toh MPHS, Leong HSS, Lim BK. Development of a diabetes registry to improve quality of care in the National Healthcare Group in Singapore. Ann Acad Med Singapore 2009; 38: 546.

48. Thambiah SC, Samsudin IN, George E, Zahari Sham SY, Lee HM, Muhamad MA, Hussei Z, Mohd Noor N, Mohamad M. Relationship between dyslipidaemia and glycaemic status in patients with type 2 diabetes mellitus. Malays J Pathol 201638 : 123-130.

49. Daousi C, Casson IF, Gill GV, MacFarlane IA, Wilding JP, Pinkney JH. Prevalence of obesity in type 2 diabetes in secondary care: association with cardiovascular risk factors. Postgrad Med J 2006; 82: 280-284. 